\title{
How Should Initial Fit Inform Soft Contact Lens Prescribing
}

Boychev Nikolay, Laughton Deborah S PhD, Bharwani Gulshan, Ghuman Hafsa, Wolffsohn James S $\mathrm{PhD}$

Ophthalmic Research Group, School of Life and Health Sciences, Aston University, Birmingham, United Kingdom

\section{Corresponding Author}

J.S. Wolffsohn, Ophthalmic Research Group, School of Life and Health Sciences, Aston University, Birmingham, United Kingdom

Email: j.s.w.wolffsohn@aston.ac.uk

Tel: +44 (0) 1212044140

Fax: +44 (0) 1212044048

\section{Abstract}

Purpose: $\quad$ To investigate how initial HEMA and silicone-hydrogel (SiHy) contact lens fit on insertion, which informs prescribing decisions, reflectend of day fit.

Methods: Thirty participants (aged $22.9 \pm 4.9$ years) were fitted contralaterally with HEMA and SiHy contact lenses. Corneal topography and tear break-up time were assessed pre-lens wear. Centration, lag, post-blink movement during up-gaze and push-up recovery speed were recorded after 5,10,20 minutes and 8 hours of contact lens wear by a digital slit-lamp biomicroscope camera, along with reported comfort. Lens fit metrics were analysed using bespoke software.

Results: $\quad$ Comfort and centration were similar with the HEMA and SiHy lenses $(p>0.05)$, but comfort decreased with time $(p<0.01)$ whereas centration remained stable $(F=0.036, p=0.991)$. Movement-on-blink and lag were greater with the HEMA than the SiHy lens $(p<0.01)$, but 
movement-on-blink decreased with time after insertion $(F=22.423, p<0.001)$ whereas lag remained stable $(F=1.967, p=0.129)$. Push-up recovery speed was similar with the HEMA and the SiHy lens 5 to 20 minutes after insertion $(p>0.05)$, but was slower with SiHy after 8 hours wear $(p=0.016)$. Lens movement on blink and push-up recovery speed was predictive of the movement after 8 hours of wear after 10 to 20 minutes SiHy wear, but 5 to 20 minutes of HEMA lens wear.

Conclusions: A HEMA or SiHy contact lens with poor movement on blink/push-up after at least 10 minutes after insertion should be rejected.

Key words: $\quad$ Contact lenses; HEMA; silicone hydrogel; settling time; prescribing 


\section{Introduction}

Dispensing a patient with well-fitting contact lenses is vital to reduce the probability of compromise to the ocular surface $[1,2]$, and to maximise ocular comfort [3,4]. Therefore it is important contact lens practitioners are able to identify whether contact lens fit is adequate during the initial fit assessment. Recent research has defined the key clinical metrics that fully describe soft lens fit [5], however soft lens fit varies with time $[2,6,7]$, requiring a settling period before lens fit stabilises, perhaps linked to changes in the post-lens tear film.[2] Contact lens movement on blink has been found previously to decrease for 30 minutes post-insertion $[2,6]$ and the optimal predictability of lens fit 8 hours post-insertion has been reported to be achieved 5 minutes post-insertion of HEMA (hydroxyethyl methacrylate) low [2] and high $[2,6]$ water content lenses. However, the temporal dependency of centration, lag and push-up recovery speed have not been quantified, and the duration of the optimum settling time for silicone hydrogel lenses prior to lens fit assessment remains equivocal. Whilst the shape profiles of HEMA and silicone hydrogel contact lenses are similar, it is feasible the higher modulus of silicone hydrogel lenses may affect the temporal characteristics of lens movement.

The aim of the current study is to objectively [8] investigate the optimum settling time of HEMA and silicone hydrogel contact lens using the full range of key clinical metrics [5] in order to develop evidence-based clinical prescribing guidance for contact lens practitioners. 


\section{Methods}

Thirty neophyte to contact lens wear participants aged $22.9 \pm 4.9$ years (10 male) were recruited following informed consent. Patients were screened to exclude those with a positive history of systemic disease, ocular disease or abnormalities (including corneal endothelial dystrophy, guttata, recurrent corneal erosion), corneal surgery, lenticular opacities, intraocular surgery, astigmatism $>0.75 \mathrm{D}$, acuity >0.0logMAR, amblyopia (>0.1 logMAR difference in visual acuity between eyes), heterotropia or anisometropia (> 1.00 D mean spherical equivalent difference between eyes). Informed written consent was obtained from all the participants after an explanation of the nature and possible consequences of the study. The study was approved by the Aston University Research Ethics Committee and conformed to the tenets of the declaration of Helsinki.

Corneal topography in primary gaze and stitched with peripheral gaze (Medmont, Nunawading, Victoria, Australia), iris diameter and non-invasive tear break-up time (average of 3 viewed with the Tearscope, Keeler, Windsor, UK) was assessed at baseline. The participants were then fitted through random assignment with a HEMA contact lens $(8.5 / 9.0 \mathrm{~mm}$ base curve Acuvue Moist, Johnson and Johnson, Jacksonville, USA) on one eye and a silicone-hydrogel contact lens $(8.4 / 8.8 \mathrm{~mm}$ base curve Acuvue Oasys, Johnson and Johnson, Jacksonville, USA) on the other (selected at random) by the same experienced investigator within the range of average keratometry readings plus 0.6 to $1.0 \mathrm{~mm}$.

At 5 minutes, 10 minutes, 20 minutes and 8 hours post-insertion, participants were asked to rate their comfort on a 0 (extreme discomfort) to 10 (couldn't feel) scale. Push-up speed of recovery was rated subjectively 63as (slow $(<2 \mathrm{~mm} / \mathrm{s}) /$ medium ( 2 to $4 \mathrm{~mm} / \mathrm{s}$ )/fast $(>4 \mathrm{~mm} / \mathrm{s}$ ) following digital displacement and centration, blink on upgaze, and horizontal lag were dynamically captured using a digital slit-lamp biomicroscope (CSO, Florence, Italy) at 6x magnification (resolution $1392 \times 1024$ pixels, frame rate $11 \mathrm{~Hz}$ ) by a different investigator ensuring each video was adequate for subsequent analysis. 
A separate masked observer objectively analysed the resulting videos (all right eyes followed by all left eyes) using a purpose-developed image analysis program (LabVIEW, National Instruments, Austin, Texas), as described previously [8]. Lens centration was determined from the difference in millimetres between the centre of circles adjusted to circumscribe the visible pupil and contact lens edge taking into account both horizontal and vertical axis. Movement on blink in up-gaze was assessed by the change in vertical lens position relative to the cornea from the first video frame after the blink until the lens was stabilised. Horizontal version lag was assessed as the difference in millimetres between the limbus to lens edge distance, from the primary gaze position to nasal- and temporal-gaze. The analysis was performed three times and the results averaged. Imaging a graticule through the same slit-lamp and camera system determined the calibration as 1 pixel being equivalent to $0.016 \mathrm{~mm}$. After analysis, the eyes were reassociated with the lens they had worn for analysis by the researcher who had conducted the randomisation.

\section{Statistical Analysis}

As the present study evaluated lens fit characteristics between techniques of assessment, rather than between eyes (a different lens type was worn in each eye), both eyes data was involved within the analysis. The Kolmogorov-Smirnov test was used to evaluate the normality of the data distribution with normally distributed data evaluated with parametric statistics. Objective data was analysed by repeated measure analysis of variance and Pearson's correlations whereas subjective data was analysed with related-samples Friedman's two-way Analysis of Variance by Ranks and Spearman rank correlations. All the videos allowed successful analysis. The results were considered statistically significant when the $p$ value was less than 0.05 . All statistical analysis was performed using SPSS for Windows statistical software (version 20, SPSS, Inc., Chicago, USA). 


\section{Results}

\section{Comfort}

Reported ocular comfort between the eye wearing the HEMA and silicone hydrogel lenses was not statistically significantly different ( $p>0.05$ at each interval), however comfort significantly decreased with time (HEMA $p<0.001$; silicone hydrogel $p=0.008$; Figure 1). Comfort after 8 hours was similarly correlated with comfort at each time period $(p<0.001)$ for the HEMA lens. The correlation with comfort at 8 hours increased with time after insertion with the silicone hydrogel lens (Table 1 ).

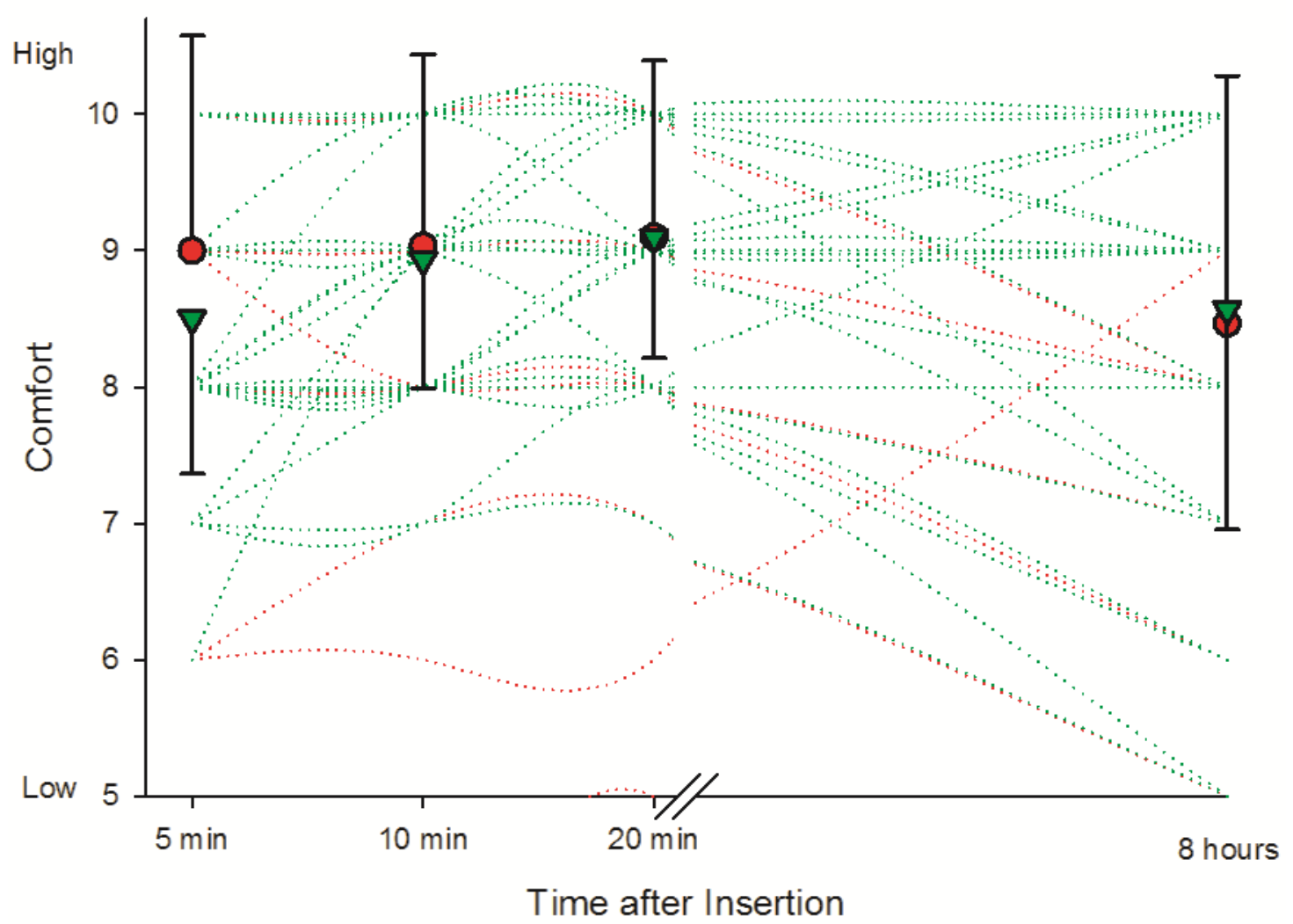

Figure 1: $\quad$ Comfort (rated 0 to 10 ) with time after insertion for HEMA (red) and silicone hydrogel (green) wearing individuals (solid lines) and on average (symbols with standard deviation error bars). $\mathrm{N}=30$ 
Table 1: The correlation coefficient of comfort, centration, blink, lag and push-up after 5, 10 and 20 minutes of HEMA and silicone hydrogel lens wear compared to after 8 hours of wear. $\mathrm{N}=30$.

\begin{tabular}{|c|c|c|c|c|c|c|}
\hline \multirow{2}{*}{$\begin{array}{l}\text { Correlation } \\
\text { with } 8 \mathrm{hrs}\end{array}$} & \multicolumn{3}{|c|}{ HEMA } & \multicolumn{3}{|c|}{ Silicone Hydrogel } \\
\hline & $5 \mathrm{~min}$ & $10 \mathrm{~min}$ & $20 \mathrm{~min}$ & $5 \min$ & $10 \mathrm{~min}$ & $20 \mathrm{~min}$ \\
\hline Comfort & $0.760 * *$ & $0.739 * *$ & $0.771 * *$ & 0.348 & $0.592^{*}$ & $0.684 * *$ \\
\hline Centration & 0.090 & 0.127 & 0.318 & 0.046 & 0.073 & 0.027 \\
\hline Blink & $0.725 * *$ & $0.723 * *$ & $0.830 * *$ & $0.732 * *$ & $0.870 * *$ & $0.900 * *$ \\
\hline Lag & $0.413 *$ & $0.699 * *$ & $0.673^{* *}$ & $0.581^{*}$ & $0.684^{* *}$ & $0.743 * *$ \\
\hline Push-up & $0.559 *$ & 0.419* & $0.530 *$ & 0.289 & $0.402^{*}$ & $0.451^{*}$ \\
\hline
\end{tabular}

$*=p<0.05 ; * *=p<0.001$

\section{Centration}

Centration was similar between the eye wearing the HEMA and silicone hydrogel lenses $(F=2.222, p$ $=0.149)$ and with time after insertion $(F=0.036, p=0.991$; Figure 2$)$, with no interaction between these factors $(F=0.901, p=0.445)$. Centration after 8 hours was not correlated with any other time period ( $p>0.05$; Table 1$)$. 


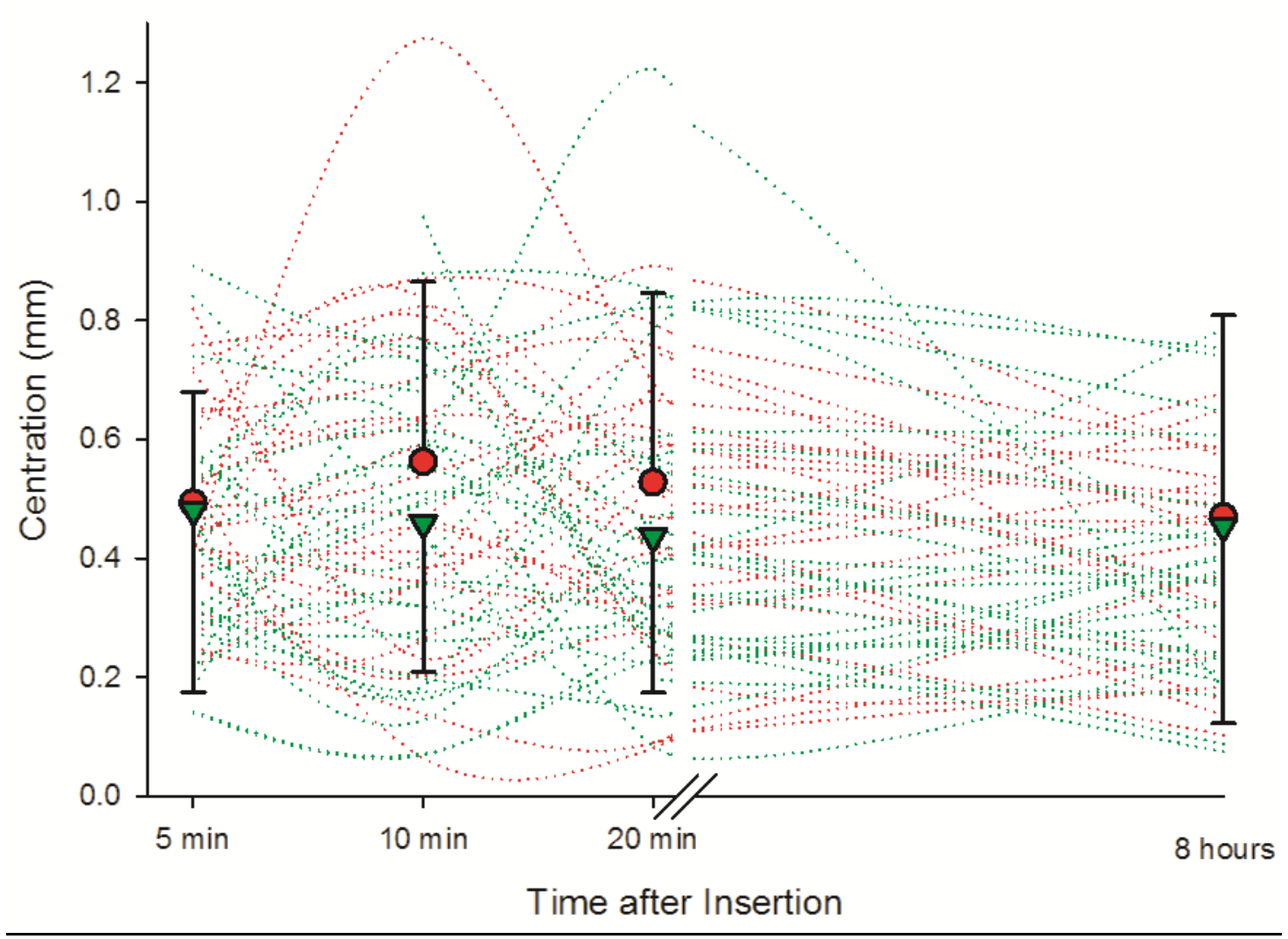

Figure 2: $\quad$ Centration with time after insertion for HEMA (red) and silicone hydrogel (green) wearing individuals (solid lines) and on average (symbols with standard deviation error bars). $\mathrm{N}=30$

\section{Movement on Blink}

Movement on blink was greater with the HEMA than the silicone hydrogel lens $(F=24.854, p<$ $0.001)$ and decreased with time after insertion $(F=22.423, p<0.001$; Figure 3$)$, although more so with the HEMA lens (interaction $F=4.094, p=0.009$ ). Movement on blink after 8 hours was correlated for each time period $(p<0.001)$ for both HEMA and silicone-hydrogel lenses, but the correlation increased with time after lens insertion (Table 1). If a movement on blink $<0.25 \mathrm{~mm}$ at 8 hours was considered unacceptable, the sensitivity and specificity was similar with assessment time 
for the HEMA lens (ROC area under the curve $5 \mathrm{~min}=0.927,10 \mathrm{~min}=0.899,20 \mathrm{~min}=0.903$ ) whereas with the silicone hydrogel it improved with time after insertion (ROC area under the curve 5 $\min =0.705,10 \min =0.828,20 \min =0.911 ;$ Figure 4).

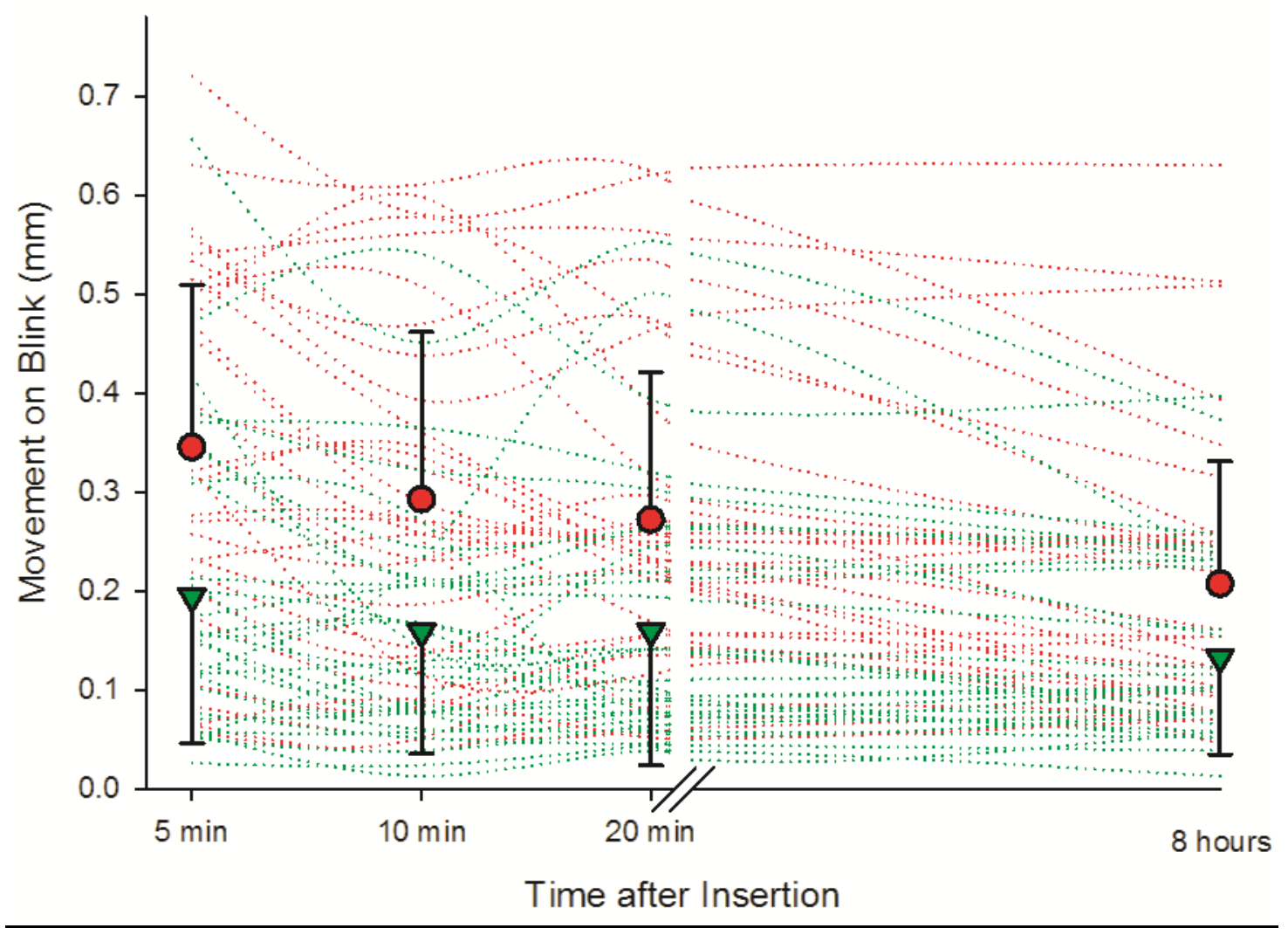

Figure 3: $\quad$ Movement on blink with time after insertion for HEMA (red) and silicone hydrogel (green) wearing individuals (solid lines) and on average (symbols with standard deviation error bars). $\mathrm{N}=30$ 


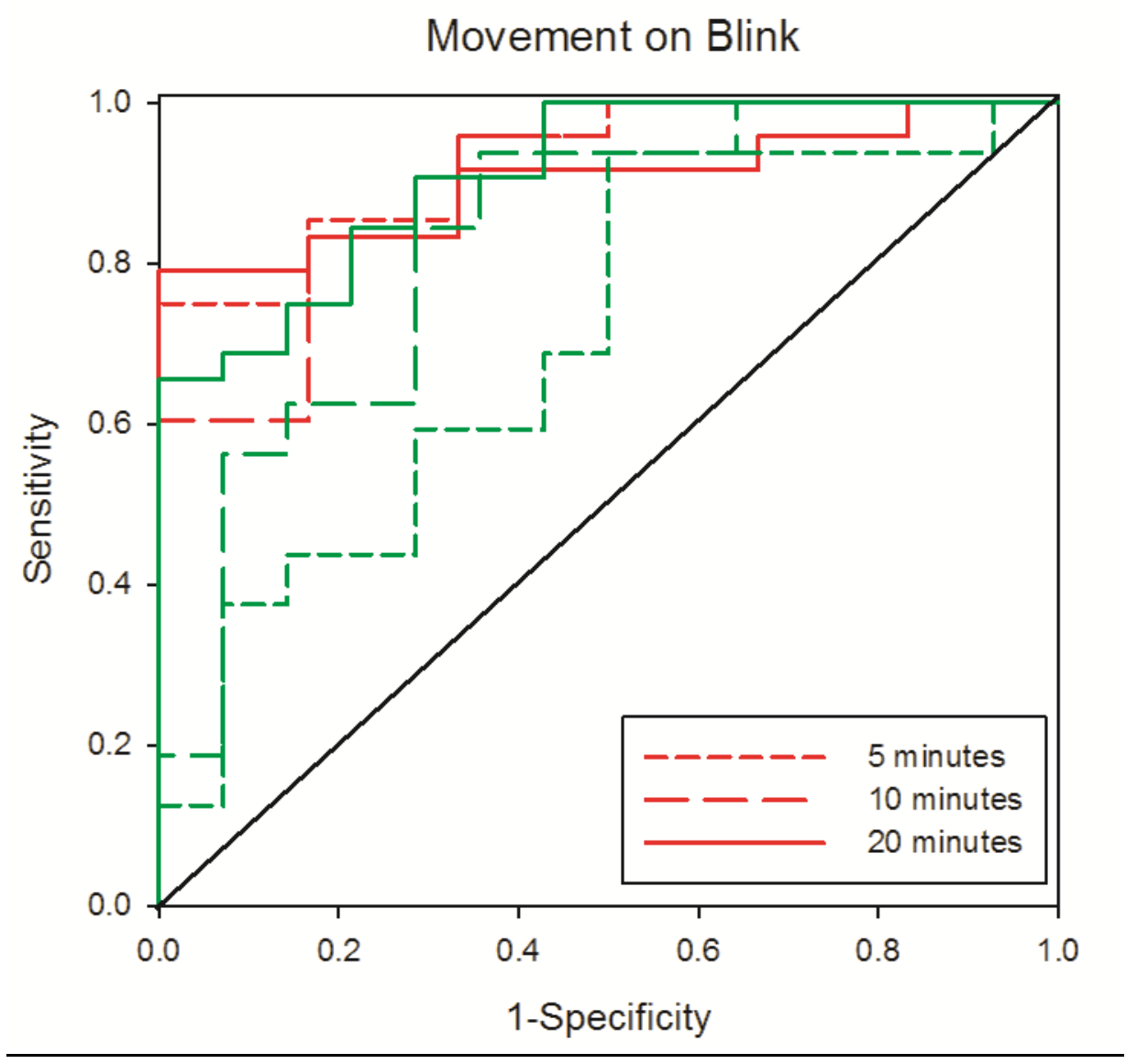

Figure 4: $\quad$ Movement on blink receiver operating characteristic curve with time after insertion for HEMA (red) and silicone hydrogel (green) wearing. $\mathrm{N}=30$

$\underline{\operatorname{Lag}}$

Lag was greater with the HEMA than the silicone hydrogel lens $(F=8.761, p=0.008)$ and remained stable with time after insertion, whereas the silicone hydrogel decreased (day (interaction $\mathrm{F}=$ 15.264, $p<0.001$; overall effect $F=1.967, p=0.129$; Figure 5). Lag after 8 hours was correlated for each time period $(p<0.05)$, but the correlation generally increased with time after lens insertion (Table 1). 


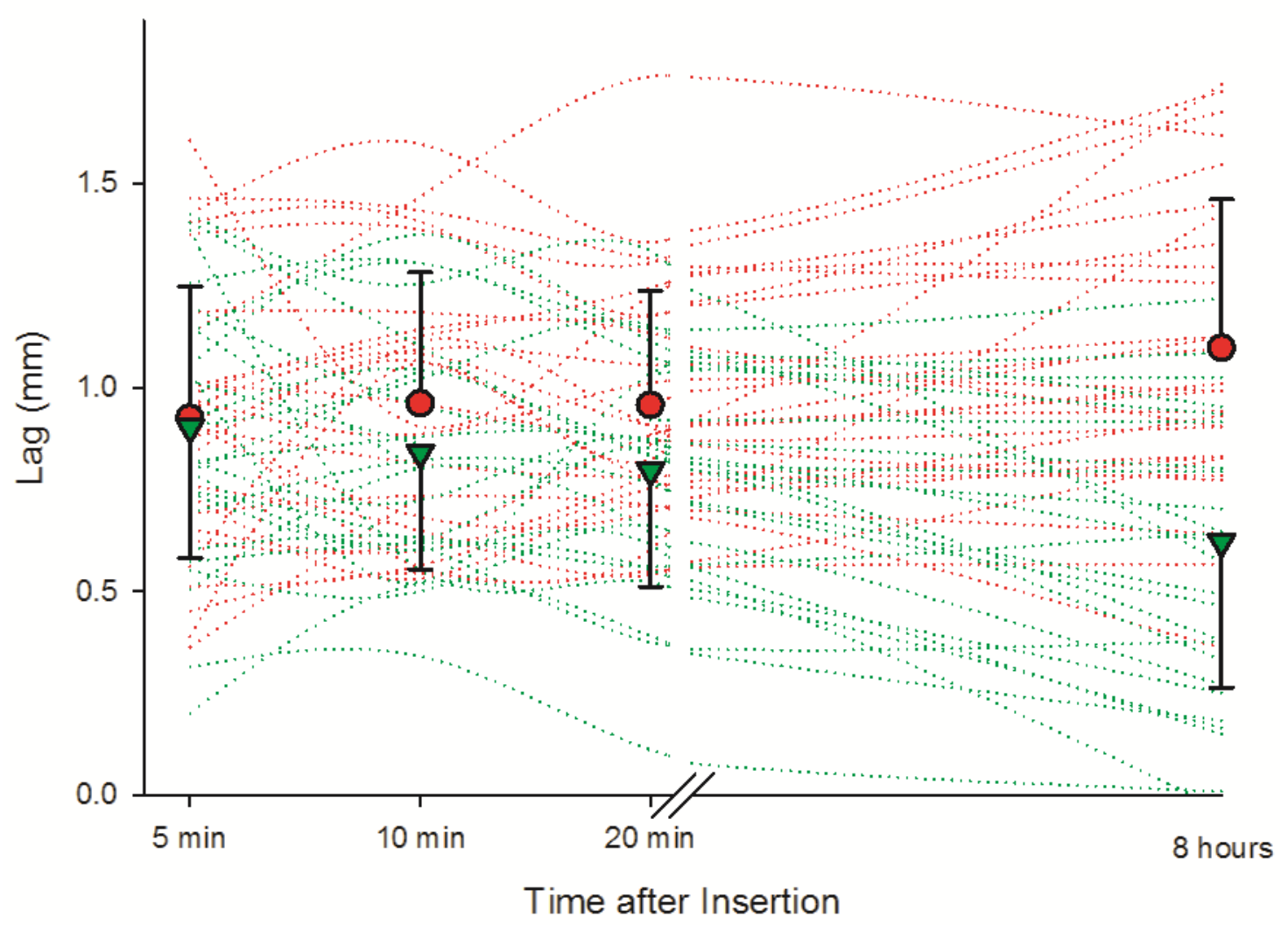

Figure 5: $\quad$ Lag with time after insertion for HEMA (red) and silicone hydrogel (green) wearing individuals (solid lines) and on average (symbols with standard deviation error bars). $\mathrm{N}=30$

\section{Push-Up Recovery Speed}

Push-up recovery speed was similar with the HEMA and silicone hydrogel lenses 5 to 20 minutes after insertion $(p>0.05)$, but was slower with silicone hydrogels than the HEMA lenses after 8 hours wear $(p=0.016$; Figure 6). Overall, push-up recovery speed was stable with time (HEMA $p=0.774$; silicone hydrogel $p=0.877$ ). Comfort after 8 hours was similarly correlated for each time period for the HEMA lens, but increase with time after insertion with the silicone hydrogel (Table 1). If a pushup speed graded as 'tight' at 8 hours was considered unacceptable, the sensitivity and specificity was slightly better at the earliest assessment time for the HEMA lens (ROC area under the curve 5 min = 
$0.846,10 \mathrm{~min}=0.726,20 \mathrm{~min}=0.726)$ whereas with the silicone hydrogel it improved with time after insertion ( $R O C$ area under the curve $5 \mathrm{~min}=0.618,10 \mathrm{~min}=0.676,20 \mathrm{~min}=0.713$; Figure 7).

There was no correlation between corneal topography (flat or steep angle, power or eccentricity and surface regularity indexes), non-invasive break-up time or lens power with comfort, centration, blink, lag or push-up recovery speed or the change in these parameters over time (taking $p<0.001$ to account for multiple correlations).

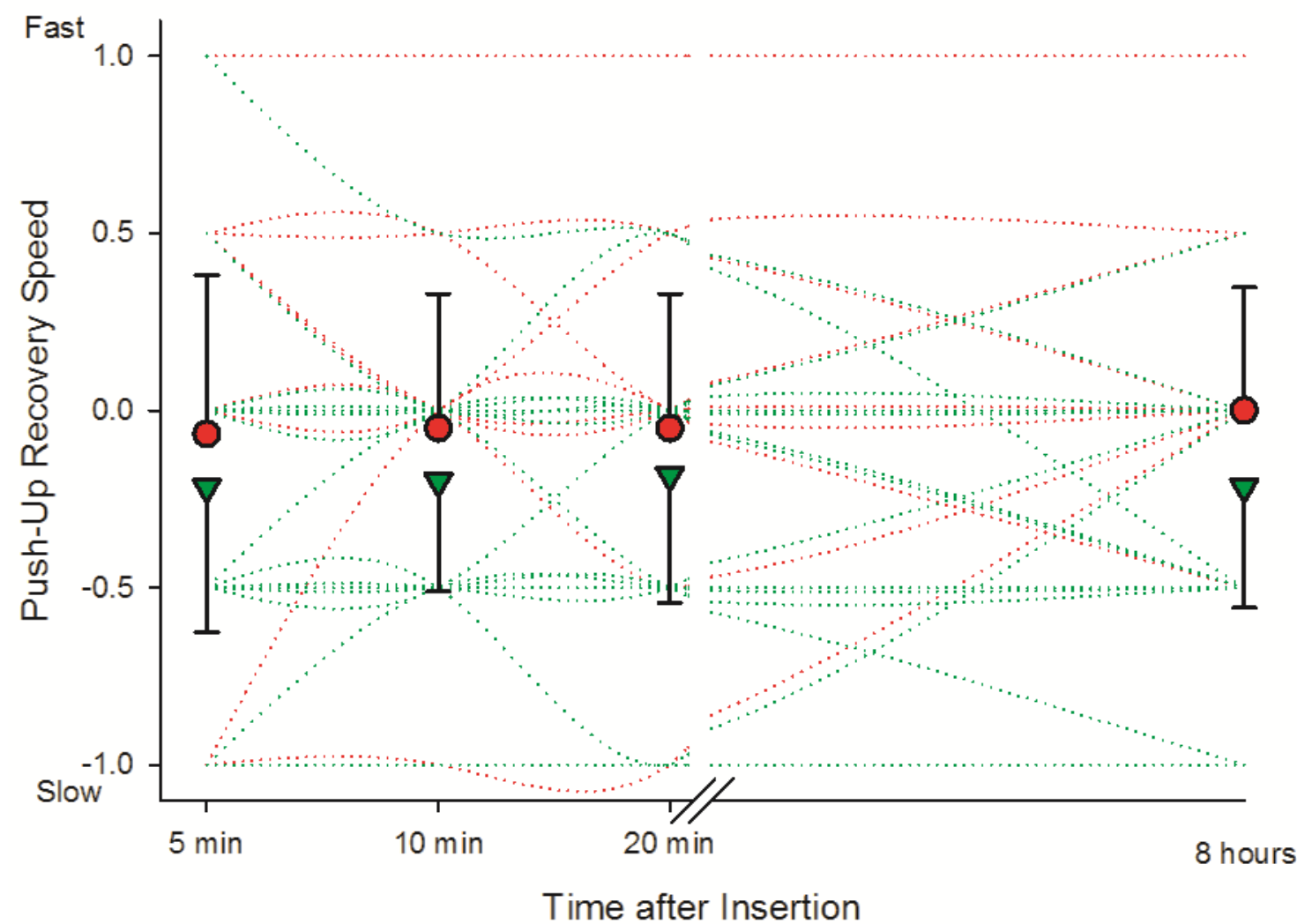

Figure 6: $\quad$ Push-up recovery speed (rated between 1 [fast] and -1 [slow] with time after insertion for HEMA (red) and silicone hydrogel (green) wearing individuals (solid lines) and on average (symbols with standard deviation error bars). $\mathrm{N}=30$ 


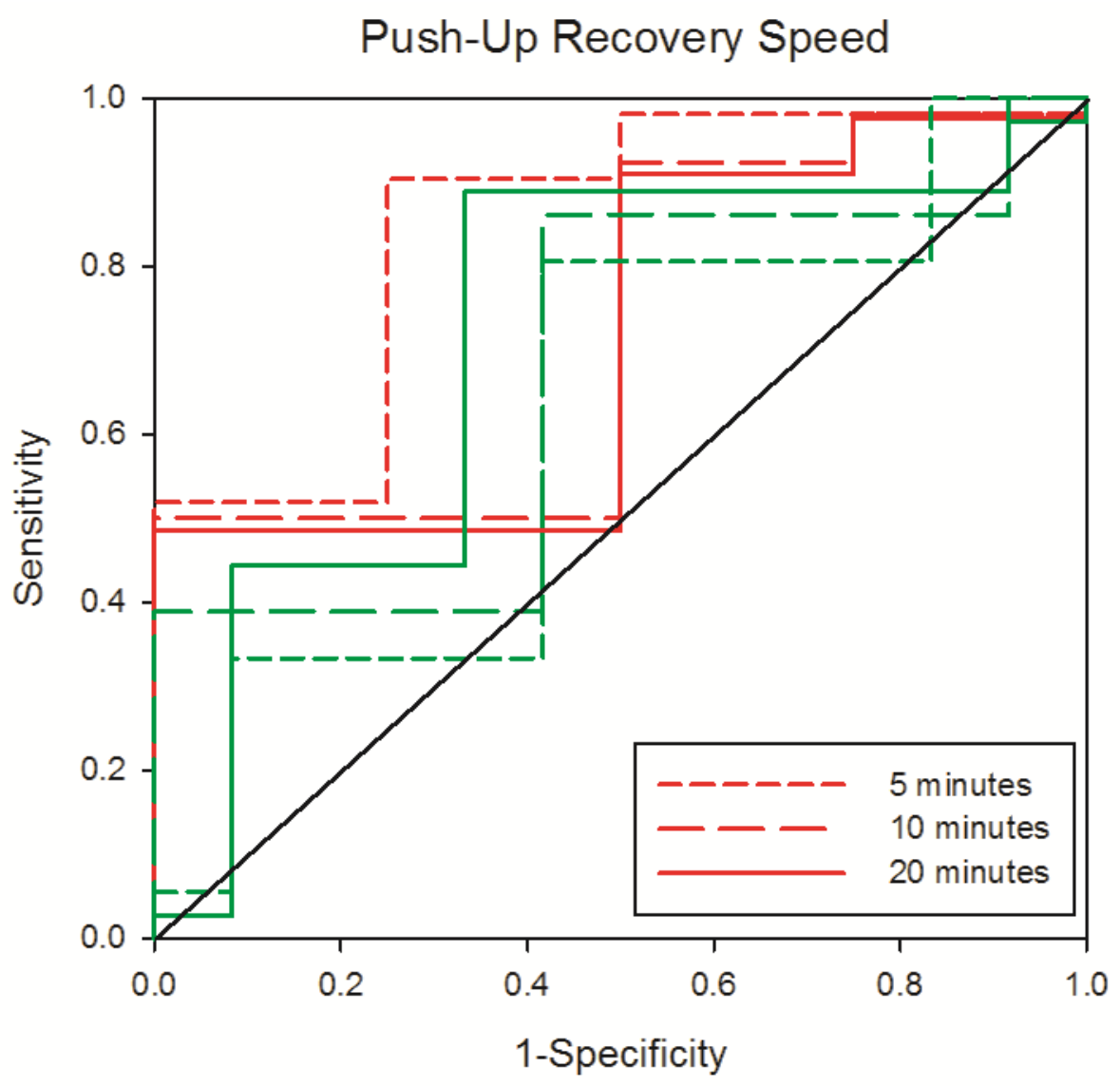

Figure 7: $\quad$ Push-up recovery speed receiver operating characteristic curve with time after insertion for HEMA (red) and silicone hydrogel (green) wearing. $\mathrm{N}=30$ 


\section{Discussion}

The current investigation is the first double-masked randomised controlled trial to investigate the optimum settling time for HEMA and silicone-hydrogel contact lenses. While only one design of each material was investigated, the design is likely to change the fit characteristics in general,[9] rather than the change in fit over time in contact lens wearers. The study was conducted on contact lens neophytes and therefore settling time might be slightly less on adapted wearers, although no subject had marked discomfort or eye watering on lens insertion. Subjects were also unrestricted in activity during the 8 hours of wear, so lens fit may be influences by environmental factors, although they were located in the clinic environment for the 15 minutes prior to the final assessment.

Similarly to previous research $[2,6]$, a decrease in lens movement on blink was observed 20 minutes after insertion of both lens types. However the biphasic relationship previously observed by Brennan et al. [2] and Schwallie and Bauman [6], where a subsequent increase in lens movement was evident after 8 hours of wear, was not observed during the present investigation with modern lens designs. The push-up recovery speed and lag of both lens types also failed to follow the aforementioned biphasic pattern with time. The reported increase in lens movement after 8 hours of wear has been suggested to be due to lens rehydration [2], however Pritchard and Fonn reported no association between lens hydration and lens movement [10]. The gradual reduction in lens movement over the course of contact lens wear is likely to be due to depletion of the post-lens tear film (PTF) with blinking [11], which has been reported to be determinant of lens movement [12]. Indeed, lens movement and PTF thickness co-vary throughout the day [12], which may create a biphasic contact lens fit profile in some individuals.

The movement of the silicone hydrogel lens on push-up, blink and lag was slower than the HEMA lens, which is contrary as to what might be expected from the stiffer material,[5] but might have resulted from the slight differences in lens design. The comfort of both lens types decreased towards the end of the day, as reported previously $[13,14]$, however no significant differences emerged 
based on lens material [15]. Movement on blink, push-up recovery speed and lag after 8 hours of wear were highly correlated to the results observed after 10 to 20 minutes of wear of the HEMA and silicone hydrogel lenses. Push-up recovery and comfort after 8 hours of silicone hydrogel lens wear was not as strongly associated with the results after 5 minutes of wear, possibly due to the initial adaptation to the higher modulus of silicone hydrogel lens materials.

If the push-up recovery speed of a lens graded as tight $\left(<2 \mathrm{~mm} / \mathrm{s}\right.$ or a $\mathrm{P}^{-}$subjective grade as proposed by the authors previously [5]) at 8 hours post-insertion was considered unacceptable, optimal speed of recovery on push-up ( 2 to $4 \mathrm{~mm} / \mathrm{s}$ ) after 5 minutes of wear was highly predictive of optimal speed of recovery after 8 hours of HEMA lens wear. Whereas, the predictive value of the speed of push-up recovery improved with time post-insertion of the silicone hydrogel lens. Similarly, if slow movement on blink ( $<0.25 \mathrm{~mm}$ or a $\mathrm{B}^{-}$subjective grade) at 8 hours post-insertion was considered unacceptable [5], the predictive value of movement on blink for optimum lens fit $(0.25$ to $0.50 \mathrm{~mm}) 8$ hours postinsertion also improved with time after insertion of the silicone hydrogel lens. The predictive value of movement on blink was similar after 5, 10 and 20 minutes of wear of the HEMA lens. These results support the work of Brennan et al. [2] and Schwallie and Bauman [6], who suggested HEMA lens fit after 5 minutes of wear was representative of lens movement after 8 hours of wear. Assessing HEMA lens fit at least 10 minutes of lens wear is also closely associated with lens fit after 8 hours of wear. However, silicone hydrogel lenses appear to take longer to settle than HEMA lenses, which is likely to be due to the higher modulus of silicone hydrogel lenses. Therefore, the optimum time to determine silicone hydrogel lens fit is possibly closer to at least 20 minutes postinsertion. Longer-term comfort, centration and lag can also be reliably assessed at this initial evaluation time although what is 'acceptable' is less evident. Future research should examine whether lens movement (such as on blink or push-up) is the best way to describe soft lens fit and what is the relevance of lens movement for example in promoting tear exchange. 
In conclusion, soft lens fit should be evaluated at least 10 minutes after lens insertion, when the findings are largely predictive of subsequent lens fit and a negative grade for movement on blink or push-up recovery [5] or poor comfort indicates the lens should be rejected.

\section{Acknowledgements}

This study was funded by a British Contact Lens Association Summer Scholarship. The authors have no other financial interest in the content of this manuscript. 


\section{References}

[1] G Young, S Coleman, Poorly fitting soft lenses affect ocular integrity, Eye Contact Lens. 27 (2001) 68-74.

[2] NA Brennan, RG Lindsay, K McCRAW, L Young, AS Bruce, TR Golding, Soft lens movement: temporal characteristics, Optom. Vis. Sci. 71 (1994) 359-363.

[3] G Young, Evaluation of soft contact lens fitting characteristics, Optom. Vis. Sci. 73 (1996) 247-254.

[4] L Jones, NA Brennan, J González-Méijome, J Lally, C Maldonado-Codina, TA Schmidt, L Subbaraman, G Young, JJ Nichols; members of the TFOS International Workshop on Contact Lens Discomfort, The TFOS International Workshop on Contact Lens Discomfort: report of the contact lens materials, design, and care subcommittee. Invest. Ophthalmol. Vis. Sci. 54 (2013) 37-70.

[5] JS Wolffsohn, OA Hunt, AK Basra, Simplified recording of soft contact lens fit, Contact Lens Ant. Eye. 32 (2009) 37-42.

[6] JD Schwallie, RE Bauman, Fitting characteristics of Dailies daily disposable hydrogel contact lenses, Eye Contact Lens. 24 (1998) 102-106.

[7] JJ Nichols, PE King-Smith, The impact of hydrogel lens settling on the thickness of the tears and contact lens, Invest. Ophthalmol. Vis. Sci. 45 (2004) 2549-2554.

[8] L Belda-Salmerón, T Drew, L Hall, JS Wolffsohn, Objective analysis of contact lens fit, Contact Lens Ant. Eye. 38 (2015) 163-167.

[9] JS Wolffsohn, T Drew, S Dhallu, A Sheppard, GJ Hofmann, M Prince, Impact of soft contact lens edge design and midperipheral lens shape on the epithelium and its indentation with lens mobility, Invest. Ophthalmol. Vis. Sci. 54 (2013) 1690-1696.

[10] N Pritchard, D Fonn, Dehydration, lens movement and dryness ratings of hydrogel contact lenses, Ophthalmic Physiol. Opt. 15 (1995) 281-286.

[11] H Kemmetmuller, Susceptibility of lacrimation to the application of contact lenses, Contact Lens J. 14 (1986) 6-9.

[12] SA Little, AS Bruce, Hydrogel (Acuvue) lens movement is influenced by the postlens tear film, Optom. Vis. Sci. 71 (1994) 364-370.

[13] D Fonn, Targeting contact lens induced dryness and discomfort: what properties will make lenses more comfortable, Optom. Vis. Sci. 84 (2007) 279-285.

[14] KA Dumbleton, CA Woods, LW Jones, D Fonn, Comfort and adaptation to silicone hydrogel lenses for daily wear, Eye Contact Lens. 34 (2008) 215-223.

[15] SW Cheung, P Cho, B Chan, C Choy, V Ng, A comparative study of biweekly disposable contact lenses: silicone hydrogel versus hydrogel, Clin. Exp. Optom. 90 (2007) 124-31. 